Review article

\title{
ADP-ribosyltransferases: plastic tools for inactivating protein and small molecular weight targets
}

\author{
Friedrich Koch-Nolte ${ }^{\mathrm{a}, *}$, Pedro Reche ${ }^{\mathrm{b}}$, Friedrich Haag ${ }^{\mathrm{a}}$, Fernando Bazan ${ }^{\mathrm{b}}$ \\ ${ }^{a}$ Institute for Immunology, University-Hospital, D20246 Hamburg, Germany \\ ${ }^{\mathrm{b}}$ DNAX Research Institute, Palo Alto, CA, USA
}

Received 13 June 2000; received in revised form 18 January 2001; accepted 25 January 2001

\begin{abstract}
ADP-ribosyltransferases (ADPRTs) form an interesting class of enyzmes with well-established roles as potent bacterial toxins and metabolic regulators. ADPRTs catalyze the transfer of the ADP-ribose moiety from NAD ${ }^{+}$onto specific substrates including proteins. ADP-ribosylation usually inactivates the function of the target. ADPRTs have become adapted to function in extra- and intracellular settings. Regulation of ADPRT activity can be mediated by ligand binding to associated regulatory domains, proteolytic cleavage, disulphide bond reduction, and association with other proteins. Crystallisation has revealed a conserved core set of elements that define an unusual minimal scaffold of the catalytic domain with remarkably plastic sequence requirements - only a single glutamic acid residue critical to catalytic activity is invariant. These inherent properties of ADPRTs suggest that the ADPRT catalytic fold is an attractive, malleable subject for protein design.
\end{abstract}

Keywords: ADP-ribosylation; Bacterial toxins; Amino acid sequence alignment; Sequence homology; Structure prediction; Protein design

\section{ADP-ribosyltransferases catalyze ADP-ribosylation of targets}

ADP-ribosyltransferases (ADPRTs) catalyze the transfer of the ADP-ribose moiety from $\beta$ nicotinamide adenine dinucleotide $\left(\mathrm{NAD}^{+}\right)$onto substrates including proteins, small molecular

\footnotetext{
* Corresponding author. Tel.: +49-40-42803-3612; fax: + 49-40-42803-4243.

E-mail address: nolte@uke.uni-hamburg.de (F. KochNolte).
}

weight substances, and water, while releasing the nicotinamide moiety (Fig. 1) (Aktories, 1991; Haag and Koch-Nolte, 1997). The ADPRTcatalysed reaction is also designated ADP-ribosylation. Some ADPRTs are promiscuous with respect to substrate specificity, e.g. several arginine-specific ADPRTs including Pseudomonas aeruginosa exoenyzme $\mathrm{S}$ and murine ART2 can ADP-ribosylate several distinct target proteins as well as simple guanidino compounds (Koch-Nolte et al., 1996a,b; Radke et al., 1999). Other AD- 
PRTs show exquisite specificity for a single target. For example, the only known substrate for the Clostridium botulinum C2 toxin and Salmonella typhimurium $\mathrm{SpvB}$ is actin, while that for diphtheria toxin and pseudomonas exotoxin $\mathrm{A}$ is elongation factor 2 (Aktories et al., 1986; Tezcan-Merdol et al., 2000; Wilson and Collier, 1992).

\section{ADP-ribosylation of proteins}

In case of protein substrates, ADPRT catalysed protein ADP-ribosylation is akin to protein kinase-catalyzed protein phosphorylation. Like phosphorylation, ADP-ribosylation is a modification, which profoundly affects protein function. Diphtheria toxin-mediated ADP-ribosylation of elongation factor 2 blocks protein synthesis while cholera toxin-mediated ADP-ribosylation of the alpha subunit of the adenylate cyclase regulating G-protein leads to chronic activation of adenylate cyclase (Aktories, 1991). Like phosphorylation, ADP-ribosylation is targeted towards specific amino acid residues. Subclasses of ADPRTs specific for arginine, cysteine, asparagine, glutamic acid, and diphthamide have been characterised. Like phosphorylation, ADP-ribosylation is potentially reversible. A distinct family of enzymes,
ADP-ribosyl-protein glycohydrolases, can remove the ADP-ribose moiety from target proteins (Moss et al., 1992), similar to the removal of phosphates from proteins by protein phosphatases. In both cases, removal of the chemical group can restore native protein function. Similar to the autophosphorylation exhibited by some protein kinases, some ADPRTs can auto-ADP-ribosylate themselves.

\section{ADP-ribosylation of small molecules}

A number of ADPRTs have been shown to ADP-ribosylate small molecules. As mentioned above, exoenzyme S and ART2 can ADP-ribosylate L-arginine in target proteins as well as free L-arginine and simple guanidino compounds. Some ADPRTs including PARP and tankyrase catalyze ADP-ribosylation of ADP-ribose itself, resulting in the formation of long, branched polymers of ADP-ribose (Oliver et al., 1999; Smith et al., 1998). Recently, a bacterial ADPRT capable of ADP-ribosylating and thereby inactivating the antibiotic rifampin was discovered (Quan et al., 1999). Moreover, a family of enzymes has been described which can transfer ADP-ribose from $\mathrm{NAD}^{+}$to a phosphate group in RNA (Spinelli et al., 1999). A number of ADPRTs can also utilise

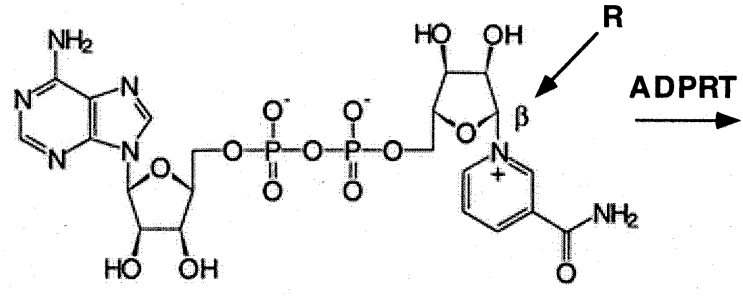

$\mathrm{NAD}^{+}+$target (R)

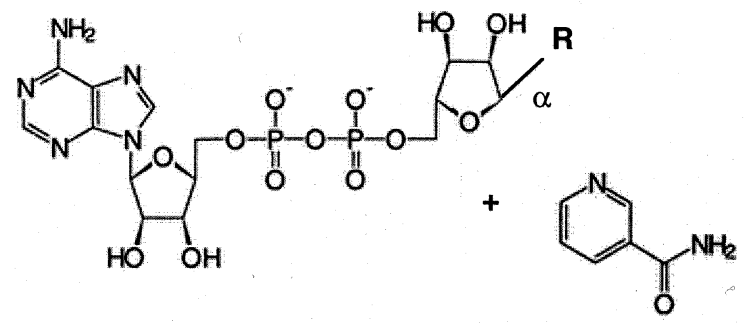

ADP-ribosylated target $+\quad$ nicotinamide

Fig. 1. Schematic diagram of the reaction catalysed by ADP-ribosyltransferases. ADP-ribosyltransferases catalyze the attack by a nucleophilic target $(\mathrm{R})$ of the $\beta$-glycosidic bond between nicotinamide and ribose in $\beta$ nicotinamide adenine dinucleotide, $\beta \mathrm{NAD}+$. This results in transfer of the ADP-ribose unit from NAD onto the target (ADP-ribosylation), while nicotinamide is released. Most commonly, the nucleophile resides in the side chain of a specific amino acid in a specific protein. ADPRTs specific for arginine, asparagine, glutamic acid, cysteine, and diphthamide (modified histidine) have been characterised by molecular cloning. Some ADPRTs can also ADP-ribosylate small molecular weight targets, e.g. the arginine-analogue agmatine or even water. The latter appears as NAD-glycohydrolase (NADase) activity. 
$\mathrm{H}_{2} \mathrm{O}$ as acceptor for ADP-ribosylation, resulting in the glycohydrolysis of $\mathrm{NAD}^{+}$to nicotinamide and ADP-ribose.

\section{Regulation of ADPRT-activity}

In the course of evolution, ADPRT catalytic domains have become associated with diverse regulatory domains (Bazan and Koch-Nolte, 1997). Some ADPRTs exhibit constitutive activity, others require proteolytic cleavage, reduction of a disulphide bond, or association with other proteins for activation. For example, diphtheria toxin is made as an inactive multidomain precursor protein (Wilson and Collier, 1992). The receptor-binding domain determines host cell specificity and the translocation domain mediates passage thrflough the cell membrane. Proteolytic cleavage unleashes the catalytic domain and reduction of a disulphide bond activates enzyme activity. The activity of mammalian nuclear poly(ADP-ribosyl)transferases PARP1 and PARP2 is regulated by binding of DNA to a linked regulatory domain (Ame et al., 1999; Oliver et al., 1999).

\section{ADPRTs function in many different cellular compartments}

ADP-ribosyltransferase has become well adapted to function in different cellular compartments. Mammalian ART1 and ART2 ADP-ribosylate cell membrane proteins on the extracellular leaflet of the plasma membrane, PARP1 and PARP2 ADPribosylate numerous nuclear proteins, and vault PARP ADP-ribosylates components of large cytoplasmic multiprotein complexes (Ame et al., 1999; Kickhoefer et al., 1999; Liu et al., 1999). Many secretory bacterial ADPRTs ADP-ribosylate cytoplasmic target proteins in animal cells (Aktories, 1991; Wilson and Collier, 1992).

\section{Topology of the catalytic core of ADPRTs}

The crystal structures of six bacterial toxin ADPRTs (see Note added in Proof) and of chicken
PARP reveal a common topology of the catalytic domain (Allured et al., 1985; Bell and Eisenberg, 1996; Choe et al., 1992; Han et al., 1999; Ruf et al., 1998; Sixma et al., 1991; Stein et al., 1994; Zhang et al., 1995). A conserved core set of six $\beta$-strands defines a minimal scaffold with remarkably plastic sequence requirements - only a single glutamic acid residue ( $\mathrm{E}$ in $\beta 5$ ) is invariant (Fig. 2) (Bazan and Koch-Nolte, 1997; Domenighini and Rappuoli, 1996; Koch-Nolte et al., 1996a,b). The importance of this residue for catalytic activity was demonstrated originally by site directed mutagenesis in diphtheria toxin (Carrol and Collier, 1984) and has hence been confirmed in all other ADPRTs thus examined (Bazan and Koch-Nolte, 1997; Domenighini and Rappuoli, 1996). Two other amino acid residues stabilise the active site and are also important for catalytic activity. These are histidine and tyrosine $(\mathrm{H}$ in $\beta 1, \mathrm{Y}$ in $\beta 2)$ in diphtheria toxin (DT), pseudomonas exotoxin A (ETA) and PARP1, but arginine and serine ( $R$ in $\beta 1$ and $S$ in $\beta 2$ ) in pertussis toxin (PT), cholera toxin (CT), $E$. coli heat labile enterotoxin (LT), and Bacillus insecticidal toxin VIP2 (Fig. 2) (Bazan and KochNolte, 1997; Domenighini and Rappuoli, 1996).

\section{Residues governing ADPRT target specificity}

The conserved topology of the active site crevice indicates that modern ADPRTs diverged from a primordial ADPRT, yielding a family of enzymes with a conserved NAD-binding fold but diverse target specificities for ADP-ribosylation (Bazan and Koch-Nolte, 1997; Domenighini and Rappuoli, 1996). The residues governing target-specificity have not yet been defined for any of the known ARTs. The exposed, nonconserved loops, which cradle the scissile glycosidic bond between ADP-ribose and nicotinamide lie in a position that faces the incoming target. These loops are highlighted in yellow, orange, and blue in the 3D structure of VIP2 in Fig. 3. Strikingly, all known arginine-specific ADPRTs contain a glutamic acid residue at the end of the loop preceeding $\beta 5$ (two residues upstream of the catalytic glutamic acid) (Fig. 2B, Fig. 3). Mutation of this residue to glutamine has been shown to convert arginine-specific ADPRT activity 
a

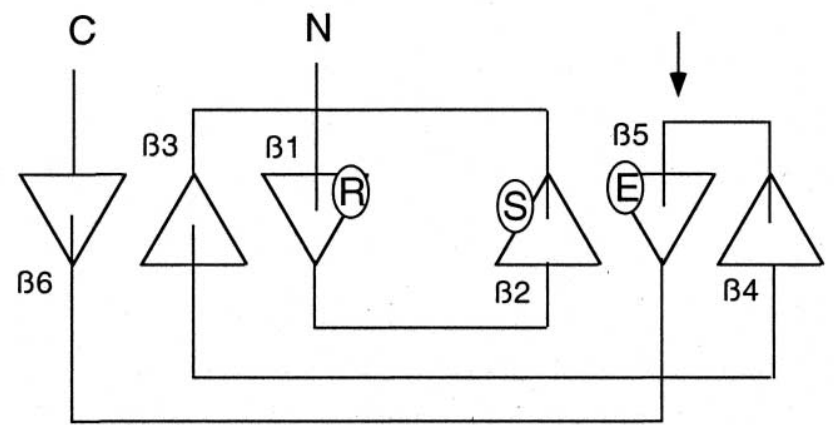

b

\begin{abstract}
PATVYRYDS 36 AFVSTSSSRRYTEVYL 18 HEIGYIYEVRADN GDRLYRADS 47 GYVSTSLSLRSAHLAG DDKLYRADS 47 GYVSTSLSLRSAHLVG NITVYRWCG 30 GYMSTSLSSERLAAFG CSLVYRGTK 14 QFSSSSLTKRVALSSN

6 YSTYYIYVIATAP

6 HSTYYIYVIATAP

1 RKIILRLOVPKGS

1 FSNHGTLFIIRTC

NESSYHGTK 26 KGEYSTDNKYDAAGYS 8 GKAGGVVKVTYPG 46 VVLSLPF VFVGYHGTF 23 RGFYIAGDPALAYGYA 10 IRNGALLRVYVPR 33 LDAITGP ROLLWHGSR 27 KGIYEADMVSKSANYC 5 DPIGLILLGEVAL 19 HSVKGLG ERMLFHGSP 22 AGIYFAENSSKSNQYV 18 ICHRQMLFCRVTL 19 HSVIGRP VRPLLHGSP 32 SGIYFSDSLSTSIKYS 5 DGTRLLLICDVAL 20 DSVHGVS
\end{abstract}

*

- NFYGAAS 22 TYOSEYLAHR 5 NIRRVTRVY

- NMENVND 8 PYEOEVSALG 5 OIYGWYRVN

- NMFNVND 8 PDEQEVSALG 5 QIYGWYRVH

- TGAYLSA 4 ASEKEILLDK 4 HIDKVTEVI

- LGVNIKE 4 PREEEVLIPG 4 HKVTAQNDN
87 PT

105 LT

61 CT

29 VIP2

53 ART2
15

434

856

1178

432
B1

B2
B3
3 SSSVEYINNW 6 SVELEINFE 3 GGRLETILGW 6 VVIPSIPTD

31 LLYNEYIVYD 4 NLKYLLKLK

4 LALAEYVIYR 4 YPEYLITYQ

B4 ß5
B6

Fig. 2. Topology cartoon (a) and structure based alignment (b) of the ADPRT catalytic core. Only the structurally conserved residues are shown. Presumptive active site residues are marked by circles in (a) and by asterisks in (b). The arrow in (a) points to the proposed loop influencing target specificity. Numbers in (b) indicate amino acid residues from the $\mathrm{N}$-and $\mathrm{C}$-terminal ends of the proteins and within connecting loops. Names of ADPRTs with known 3D structures are in boldface and their respective $\beta$ sheet residues are underlined. Sequences were compiled from database accession numbers: P04977: pertussis toxin (PT); P43530: E. coli heat labile enterotoxin (LT); D30052: cholera toxin (CT); 1QS2: Bacillus cereus insecticidal toxin (VIP2); X87612: mouse ecto-ADP-ribosyltransferase (ART2); X00703: diphtheria toxin (DT); P11439: Pseudomonas aeruginosa exotoxin A (ETA); P26446: chicken poly-ADP-ribose-polymerase (PARP); AF082556: human tankyrase (Tankyr); and AF158255: human vault-PARP (vPARP).

into NAD-glycohydrolase activity in case of Clostridium botulinum C2 toxin, Clostridium perfringens iota toxin, Pseudomonas exoenyzme S, and rat ART2 (Barth et al., 1998; Hara et al., 1996; Karsten et al., 1997; Nagahama et al., 2000; Radke et al., 1999). PARP contains a large insertion relative to other ADPRTs preceding $\beta 5$ and this region has been implicated in binding the ADP-ribose polymer target (Ruf et al., 1998).

\section{Designer ADPRTs}

With the three-dimensional structures of $\mathrm{kn}$ - own ADPRTs as a guide, it should be possible to imitate nature and to alter the target specificity of ADPRTs by mutagenesis. Transforming existing ADPRTs into one another by site directed mutagenesis and grafting of specificity loops should be relatively straight forward with more closely related family members, but poses a greater challenge for more distant relatives. The fruit of such endeavours will be a better understanding of the structure/function relationship of ADPRT target specificity. With insight into the molecular basis of ADPRT target specificity it may be even possible one day to redirect ADPRTs from known to novel targets. Examples 
a

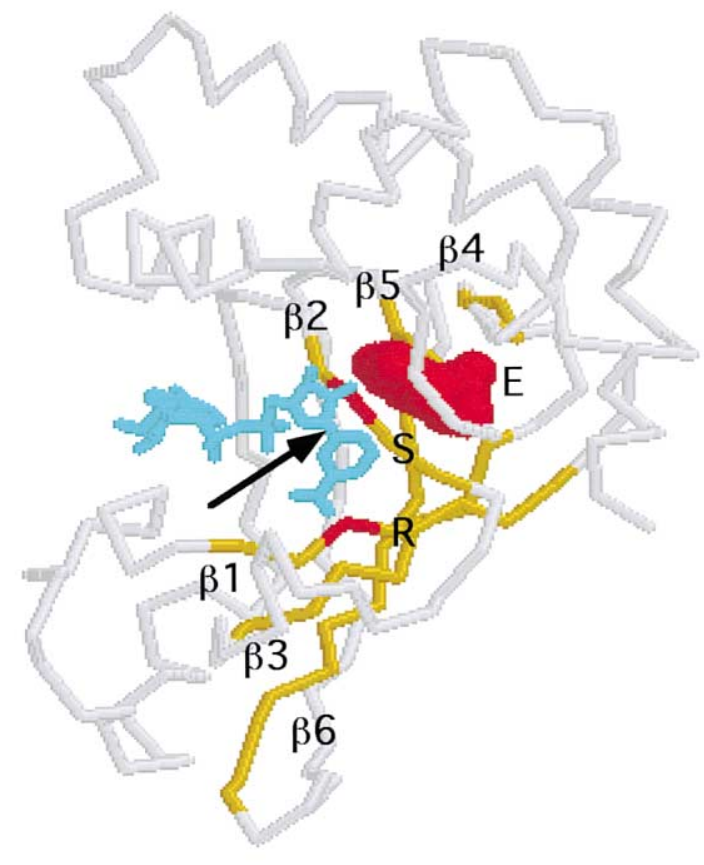

b

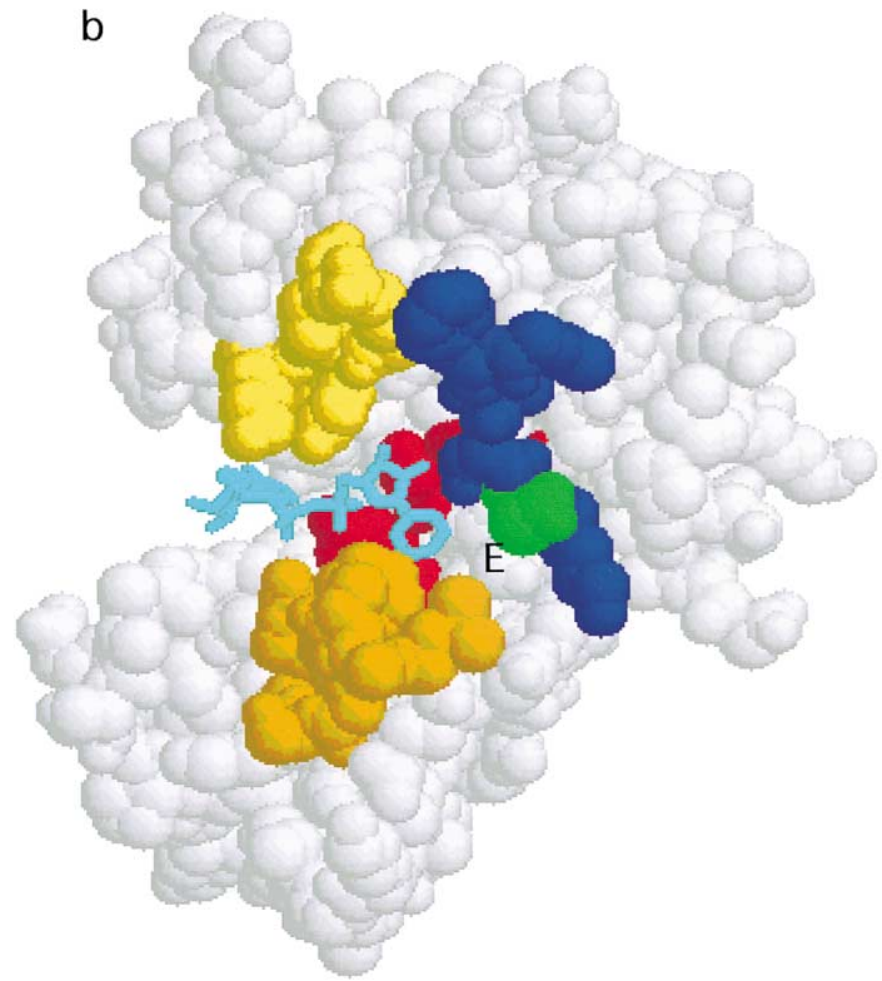

Fig. 3. Polypeptide backbone (a) and space-fill model (b) of VIP2, an actin-specific ADPRT from Bacillus cereus (Han et al., 1999). (a) The six beta sheets forming the active site crevice are highlighted in yellow and are labelled as in Fig. 2 . The active-site residues arginine R349, serine S386, and glutamic acid E428 are depicted in red and correspond to the residues marked by asterisks in Fig. 2. The only side chain shown is that of E428. The substrate NAD is depicted in cyan as a stick-model, the arrow points to the scissile glycosidic bond. (b) Space-fill model of VIP2 is depicted from the same angle as in (a). The active site residues R349, S386 and E428 are in red. The three loops that cradle the scissile glycosidic bond in NAD are highlighted in yellow, orange, and blue. The glutamic acid residue E426 in the loop preceding $\beta 5$ which is characteristic of arginine-specific ADPRTs is depicted in green. The figure was compiled with the Rasmac program using the co-ordinates for Bacillus cereus insecticidal toxin VIP2 (PDB accession number 1QS2).

for practical applications that we envision include redirecting an ADPRT from fibroblast growth factor or tuftsin (Jones and Baird, 1997; Terashima et al., 1997) to other biologically important signalling peptides; from rifampin (Quan et al., 1999) to another antibiotic, or from one nucleotide-binding protein (EF2, actin, G-alpha, rho, ras) to another key metabolic regulator. Known ADPRTs have already proven their manifold usefulness as experimental tools in cell biology, e.g. to inhibit protein synthesis (ETA, DT), cytoskeletal functions (C2, C3), signal transduction (CT, PT), T-cell activation (ART1, ART2) (Aktories, 1991; Haag and Koch-Nolte, 1997; Koch-Nolte et al., 1996a,b; Liu et al., 1999;
Wilson and Collier, 1992). It is not hard to imagine that novel designer ADPRTs would be useful in these and other settings. The properties of natural ADPRTs - their plasticity in amino acid sequence requirements, their diverse target specificities, and their propensity to function in different environments-suggest that the ADPRT fold represents an attractive, malleable, and well suited subject for protein design.

\section{Note added in proof}

In their recent paper (Han et al., 2001) describing the $3 \mathrm{D}$ structure of the $\mathrm{C} 3$ exonenzyme from 
Clostridium botulinum, Tainer and coworkers also point out the importance of the bipartite loop connecting $\beta 4$ and $\beta 5$ for substrate recognition specificity (i.e. the loop marked by the arrow in Fig. 2 and highlighted in blue in Fig. 3.

\section{Acknowledgements}

This work was supported in part by DFG-grant No310 to FKN. DNAX is fully supported by Schering-Plough Corporation.

\section{References}

Aktories, K., 1991. ADP-Ribosylating Toxins. Springer, Berlin.

Aktories, K., Barmann, M., Ohishi, I., Tsuyama, S., Jakobs, K.H., Habermann, E., 1986. Botulinum C2 toxin ADP-ribosylates actin. Nature 322, 390-392.

Allured, V.S., Collier, R.J., Carrol, S.F., McKay, D.B., 1985. Structure of exotoxin A of Pseudomonas aeruginosa at 3.0 angstrom resolution. Proc. Natl. Acad. Sci. USA 83, $1320-1324$.

Ame, J.C., Rolli, V., Schreiber, V., Niedergang, C., Apiou, F., Decker, P., Muller, S., Hoger, T., Menissier-de Murcia, J., de Murcia, G., 1999. PARP-2, A novel mammalian DNA damage-dependent poly(ADP-ribose) polymerase. J. Biol. Chem. 274, 17860-17868.

Barth, H., Preiss, J.C., Hofmann, F., Aktories, K., 1998. Characterization of the catalytic site of the ADP-ribosyltransferase Clostridium botulinum $\mathrm{C} 2$ toxin by site-directed mutagenesis. J. Biol. Chem. 273, 29506-29511.

Bazan, J.F., Koch-Nolte, F., 1997. Sequence and structural links between distant ADP-ribosyltransferase families. Adv. Exp. Med. Biol. 419, 99-107.

Bell, C.E., Eisenberg, D., 1996. Crystal structure of diphtheria toxin bound to nicotinamide adenine dinucleotide. Biochemistry $35,1137-1149$.

Carrol, S.F., Collier, R.J., 1984. NAD + binding site of diphtheria toxin: identification of a residue within the nicotinamide subsite by photochemical modification with NAD. Proc. Natl. Acad. Sci. USA 81, 3307-3311.

Choe, S., Bennett, M.J., Fujii, G., Curmi, P.M., Kantardjieff, K.A., Collier, R.J., Eisenberg, D., 1992. The crystal structure of diphtheria toxin. Nature 357, 216-222.

Domenighini, M., Rappuoli, R., 1996. Three conserved consensus sequences identify the NAD-binding site of ADP-ribosylating enzymes, expressed by eukaryotes, bacteria and T-even bacteriophages. Mol. Microbiol. 21, 667-674.

Haag, F., Koch-Nolte, F., 1997. ADP-Ribosylation in Animal Tissues: Structure, Function and Biology of Mono(ADPRibosyl)transferases and Related Enzymes. Plenum Press, New York.
Han, S., Craig, J.A., Putnam, C.D., Carozzi, N.B., Tainer, J.A., 1999. Evolution and mechanism from structures of an ADP-ribosylating toxin and NAD complex. Nat. Struct. Biol. 6, 932-936.

Han, S., Arvai, A., Clancy, S., Tainer, J.A., 2001. Crystal structure and novel recognition motif and Rho ADP-ribosylating C3 exoenzyme from Clostridium botulinum: structural insights for recognition specificity and catalysis. J. Mol. Biol. 305, 95-107.

Hara, N., Tsuchiya, M., Shimoyama, M., 1996. Glutamic acid 207 in rodent T-cell RT6 antigens is essential for argininespecific ADP-ribosylation. J. Biol. Chem. 271, 2955229555.

Jones, E.M., Baird, A., 1997. Cell-surface ADP-ribosylation of fibroblast growth factor-2 by an arginine-specific ADP-ribosyltransferase. Biochem. J. 323, 173-177.

Karsten, S., Schröder, J., da Silva, C., Kahlke, D., Thiele, H.G., Koch-Nolte, F., Haag, F., 1997. Expression and comparative analysis of recombinant rat and mouse RT6 T cell mono(ADP-ribosyl)transferases in E. coli. Adv. Exp. Med. Biol. 419, 175-180.

Kickhoefer, V.A., Siva, A.C., Kedersha, N.L., Inman, E.M., Ruland, C., Streuli, M., Rome, L.H., 1999. The 193-kD vault protein, VPARP, is a novel poly(ADP-ribose) polymerase. J. Cell. Biol. 146, 917-928.

Koch-Nolte, F., Haag, F., Kastelein, R., Bazan, F., 1996 a. Uncovered: the family relationship of a T-cell-membrane protein and bacterial toxins. Immunol. Today 17, 402-405.

Koch-Nolte, F., Petersen, D., Balasubramanian, S., Haag, F., Kahlke, D., Willer, T., Kastelein, R., Bazan, F., Thiele, H.G., 1996b. Mouse T cell membrane proteins Rt6-1 and Rt6-2 are arginine/protein mono(ADPribosyl)transferases and share secondary structure motifs with ADP-ribosylating bacterial toxins. J. Biol. Chem. 271, 7686-7693.

Liu, Z.X., Yu, Y., Dennert, G., 1999. A cell surface ADP-ribosyltransferase modulates $\mathrm{T}$ cell receptor association and signalling. J. Biol. Chem. 274, 17399-17401.

Moss, J., Stanley, S.J., Nightingale, M.S., Murtagh, J.J., Monaco, L., Mishima, K., Chen, H.C., Williamson, K.C., Tsai, S.C., 1992. Molecular and immunological characterization of ADP-ribosylarginine hydrolases. J. Biol. Chem. 267, 10481-10488.

Nagahama, M., Sakaguchi, Y., Kobayashi, K., Ochi, S., Sakurai, J., 2000. Characterization of the enzymatic component of Clostridium perfringens iota-toxin. J. Bacteriol. 182, 2096-2103.

Oliver, F.J., Menissier-de Murcia, J., de Murcia, G., 1999. Poly(ADP-ribose) polymerase in the cellular response to DNA damage, apoptosis, and disease. Am. J. Hum. Genet. 64, 1282-1288.

Quan, S., Imai, T., Mikami, Y., Yazawa, K., Dabbs, E.R., Morisaki, N., Iwasaki, S., Hashimoto, Y., Furihata, K., 1999. ADP-ribosylation as an intermediate step in inactivation of rifampin by a mycobacterial gene. Antimicrob. Agents Chemother. 43, 181-184. 
Radke, J., Pederson, K.J., Barbieri, J.T., 1999. Pseudomonas aeruginosa exoenzyme $\mathrm{S}$ is a biglutamic acid ADP-ribosyltransferase. Infect. Immun. 67, 1508-1510.

Ruf, A., Rolli, V., de Murcia, G., Schulz, G.E., 1998. The mechanism of the elongation and branching reaction of poly(ADP-ribose) polymerase as derived from crystal structures and mutagenesis. J. Mol. Biol. 278, 57-65.

Sixma, T.K., Pronk, S.E., Kalk, K.H., Wartna, E.S., Van, Z.B., Witholt, B., Hol, W.G., 1991. Crystal structure of a cholera toxin-related heat-labile enterotoxin from E. coli. Nature 351, 371-377.

Smith, S., Giriat, I., Schmitt, A., de Lange, T., 1998. Tankyrase, a poly(ADP-ribose) polymerase at human telomeres. Science 282, 1484-1487.

Spinelli, S.L., Kierzek, R., Turner, D.H., Phizicky, E.M., 1999. Transient ADP-ribosylation of a 2'-phosphate implicated in its removal from ligated tRNA during splicing in yeast. J. Biol. Chem. 274, 2637-2644.

Stein, P.E., Boodhoo, A., Armstrong, G.D., Cockle, S.A.,
Klein, M.H., Read, R.J., 1994. The crystal structure of pertussis toxin. Structure 2, 45-57.

Terashima, M., Hara, N., Badruzzaman, M., Shimoyama, M., Tsuchiya, M., 1997. ADP-ribosylation of tuftsin suppresses its receptor-binding capacity and phagocytosis-stimulating activity to murine peritoneal macrophages. FEBS Lett. 412, 227-232.

Tezcan-Merdol, D., Nyman, T., Lindberg, U., Haag, F., Koch-Nolte, F., Rhen, M., 2000. The Salmonella enterica virulence protein SPVB mediates ADP-ribosylation of actin. Mol. Microbiol. 39, 606-619.

Wilson, B.A., Collier, R.J., 1992. Diphtheria toxin and Pseudomonas aeruginosa exotoxin A: active-site structure and enzymic mechanism. Curr. Top. Microbiol. Immunol. 175, 27-41.

Zhang, R.G., Scott, D.L., Westbrook, M.L., Nance, S., Spangler, B.D., Shipley, G.G., Westbrook, E.M., 1995. The three-dimensional crystal structure of cholera toxin. J. Mol. Biol. 251, 563-573. 